\title{
Existence Fabrics
}

\author{
Isai Mathias $^{1}$ \\ ${ }^{1}$ Affiliation not available
}

June 2, 2020

Why is there something rather than nothing? It is impossible to imagine zero existence. We find ourselves on this ball known as Earth that is floating in a vast box. As one who faints only to wake at a nearby hospital, we start asking questions. Of all the inventions of humankind, science most passionately attempts to answer that Great Questionnaire, of which philosophy is its principle contributor. But there are many things we found in our ball and box too that still do not make sense. And by that I mean, there is enormous ground for unrestricted opinions due to lack of consistent truths. That is not necessarily a detour in our quest, some problems are more complex than others. We may not expect a consensus on what existence-in-itself means the same way we can prove the sun sets in the West.

To most people, life and existence are synonyms. Biology has historically studied the living and Physics the dead world. This distinction grows faint with the emerging field of Biophysics. By using the same laws to explain both worlds, a collective paradigm of everything may be reached. This work is based on such grounds.

\section{A Protest for One Fabric}

Species are proverbially defined of their structural organization. Biophysical organs conspire to certain expectation range beyond which the whole can not function. Ants don't walk a mile. Man has through biology the tools for exercising dominion, which is the only purpose for meaning. In the likeness and image of The Creator, appreciable levels of omniscience, omnipotence and Agape are attainable. This is the peculiarity of humans. Unlike other higher consciousness beings, only Man has an afterlife. The course of events on Earth transformed, actually stooped Nature, so that a renewal is necessitated. All extants, including God - by virtue of transcendence - can be on space-time. Now, presence is not the same as liveliness. The former is the common notion of existence in statements the manner of "though shalt not exist but live." Note that Creation really ended. The afterlife(if any) is mere revision to an older version, the original 6 day form. Even procreation follows already defined architectures. The biologist understands meiosis and the hereditary mechanisms and much of cellular-level activity is random. Sure, but do we know enough to determine the programming languages and algorithm of life? Contrary to popular belief, space-time is the only fabric of existence. For Eden and Heaven are synonymous, we may not find early life inhabiting elsewhere. All realities occur on space and time simultaneously. The latter sometimes protrudes to everlastingness, a time-sensitive/time-bound notion of eternity. The former, as some theories show may exceed known dimensions, namely length breadth and height. Could The Fall lead to a gradual decline in fabric quality as seen in human condition? Rather not, that would be re-Creation, linguistically feasible but unnecessary for Creation was a finite event. The goodness factor is lowered only perspectival to humankind, other objects bear their Ideality, at least when not interacting with Man. Which is a slime chance given the matter-wave commonality between things.

So, what is special about humankind? Our space-time is not exclusively experienced. This very moment an enormous population of spiritual agents traverse unperceived. Our biology, highly shared. From the totality of beings, is our Edenic intelligence superseded by any? As in any significance ratio, specialty is a function 
of the one and only. Cosmic loneliness theories suggest, of the trillions of planets in the observable universe, only Earth contains a biological essence. Thus from a realization of the vast space(and time) available for humankind (and fellow Earthlings) uniqueness arises. (Contra: how would this elation shift when no would die, and we are populous throughout the cosmos? In Heaven there are cities and therefore communities. How vast the habitats? A universe for everyone, to create and dominate or experience?).

\section{DUALISM}

After Descartes the idea of separate worlds has received little coverage. Dual realities are necessitated by the fact of existence. We know that an architect precedes the civil engineer. In this same manner, the Mind(here a representative of Human Trinity, Her spiritual realm. It follows that biology through the human body is not special at all. Derived from earth thus stardust, majority of land animals have this skin too. With trivial genomic differences it is susceptible to focus on neural patterns and hope to set Man aside.

We may suppose that existence is not inherently biological at least on the premise of supernaturalism. Here the concept of Heaven would suffice as a spiritual construct unless angels (and other beings there) have shape and concrete forms. Now at Eden, the earliest space-time for biology, Man still conversed with these hierarchical fellows in Heaven. Such communications require a spirituality. Another interesting idea is the inner talk. Apart from being the language of cognitive processing, some subtle voice in every Man persists. Fatal conditions are reached when this transcendental self is intentionally or behaviorally silenced by external influences. The body thus controls the spirit, an impossible reversal that does no good, actually all organismal function is trivial in purposes.

Human Trinity consists of Spirit, Soul and Flesh. Spirit, the literal construct of Person. Qualities that inadvertently define function reside here. Major compartments are Mind(realized via nervous system) Heart(realized via hormonal secretions) and Will(realized via intentionality protocols as choice or the decision-making process. Soul, the inducer of eternal presence. Connected to The Source of Life is the substrate of extantism. All renewal processes, all organisms, all substances are built to ever last. This is the link. Flesh, here frequently referred to as biology is the most pronounced subset of Man. Hosting discernible features allowing interactivity with environments and other extants alike. These three combined form a trichotomy we call Human Being. Which even linguistically arrives us to a dualistic conclusion. Human implies biological organization. And we already know that all beings are non-physical. From this standpoint we are spiritual-physical creatures. Other matter may be redesigned for resilience such that their continued existence is not due to soul rather master engineering. We know of rocks and trees with lifespans beyond the thousands.

If reality is not dual Physics would have been the first to prove the existence of God(and other so-called supernaturals). For they all would be here, physical, discernible and directly interactive. That is not the case. Transcendence is only possible because there are two worlds equally on the same fabric. One implements the other. This very moment I'm alive since Soul is still linked to Flesh my brain receives commands from my Mind(and not vice versa as the neuroscientist would have it) allowing me write this text. We then discuss how this operates on a practical scale.

Supposedly neuronal ions respond to certain field frequencies of Mind. By entanglement simultaneously, instantaneously the outputs of one's spirit are realized on their biological framework thus the realexternal world. For all physicality is matter and energy as propagates by field forces; spiritual content would have no trouble setting resonance networks for one way information transfer. Therefore perception too stems from Heartsense so that subjectivity and phenomenality attaches to contextual experiences in our immediate environment. Thus multitudinous, actually infinite relations concurrent with the spiritual realm are discretely portrayed in the universe, one embodied spirit after another. Beyond binary creatures, humankind including their universe are dual extants, period. What is effective than having both sides? What a pleasure to be a species of twoworlds and yet contain unparalleled oneity? What an existence!

\section{THOUGHTS \& MOMENTS}


Nature is known for its discreteness such that all flows actually come in quantities. As photon is to light, the quanta of thought is the thoughtbit. This fundamentality combines experiential data sets in a way organismal function is exercised. Its widest application is setting up what I call a Momentverse. An intentional conscious extant space coupled with a thoughtbit rather than time. Now this is utterly non-Einstenian but upon analysis the thoughtbit seldom contains horological data namely day or night and time of day for a task. Abbreviated as tbit, this cognitive quantum has significant applications to memory. Let say, Hunches do not come in full layout, as the name suggests they are mere hints, eureka if you may. To remember such random insight one might conjecture a tbit by analysing their immediate environment, internally and externally, associating them to prior information to create a concrete superstructure, unique and individualistic to them, that guarantee permanence in long term memory. Also thoughtbit technology is essential in training nonhuman sentient beings such as synthetic artificial intelligences. Allowing organization of big data into definite tbits accelerates learning whilst minimizing power consumption due to multitudinous parallel processing. Thoughtbits are how the brain encodes data, in its usual economics a derivation thereof provides information necessary to run a momentverse. In this manner tbits are building blocks of our holographic universe and mimetic world.

\section{MEANING}

Purpose is essential as a limiting factor for intrinsic ability. Organismic expression does not permate unboundedly. Living a meaningful life involves the pursuit of singular works, as is Creation, the act that distinguished Jehovah, our Creator. We too need to meet ends whose aesthetics sufficiently lasts to eternity. Unforgettable designs during temporality that transcend this version of space-time. One sure way to get there is by evading mainstream. For in uniqueness not commonality lies potential. Doing what others wouldn't be done is the crown of significance. When our lives are unparalleled i.e non-relativistic to other agents then we are important not just another "thing". Now this is the essence of existence fabrics. 